\title{
A Classical Methodology of AES Algorithm using Cipher Key
}

\author{
Laxmi Palamarthi, C.Murali Krishna
}

\begin{abstract}
Expanding might want information insurance in PC systems diode to the occasion of numerous cryptanalytic calculations hence causing information immovably over a transmission connect is basically vital in a few applications. Equipment usage of cryptanalytic calculations square measure physically secure than bundle executions since outside aggressors can't change them. So as to accomplish better in the present intensely stacked correspondence systems, equipment execution could be an insightful choice as far as higher speed and dependableness. In This paper shows the gear utilization of Advanced cryptography dynamic (AES) rule abuse Xilinx-virtex five Field Programmable Gate Array .In solicitation to achieve higher tempo and lesser freedom Sub PC memory unit movement, Inverse Sub PC mu action, join Column action and Inverse solidify Column assignments square measure arranged as LUTs and Read Only Memorie.
\end{abstract}

\section{Keywords-FPGA, LUT, ROM. AES}

\section{INTRODUCTION}

Cryptography's connects with people to proceed with the conviction determined in to the physical world to a de worlds. The centrality of cryptography's is relentlessly creating since the degree of delicate data organism transmit over an open domain is besides widening a small piece at once. The more data that is transmit in PC fathomable structure, the extra feeble we happen to to motorized spying. Cryptography's isn't only essential in guarantee application yet besides crucial in certifiable applications, for instance, E-exchange, E-mail, etc.

The AES was dissipated by National Institute of principles and Technology in 2001. Later Rijndael figuring would picked as AES check. Rijndael estimation can has key extent of 128,192 and 256 bits while square size 128 piece.

\section{ADVANCED ENCRYPTION STANDARD:}

The AES is a PC safety dynamic from NIST proposed for ensuring dc information. The AES cryptography tally is set up for encoding and unraveling 128 piece information utilizing figure encryption is having four exercises

1.Substitution $\mathrm{s}$

2. Move Row

3. Blend Columns

4. Key Additions
Revised Manuscript Received on December 12, 2019

Laxmi Palamarthi, Asst.Prof.Department of ECE,Malla Reddy Engineering College for women,Maisammaguda, Secunderabad,Telangana,India.E-mail:mkn20679@gmail.com.

C.Murali Krishna , Asst.Prof.Department of ECE,Malla Reddy Engineering College for women,Maisammaguda, Secunderabad,Telangana,India.E-mail:mkn20679@gmail.com.

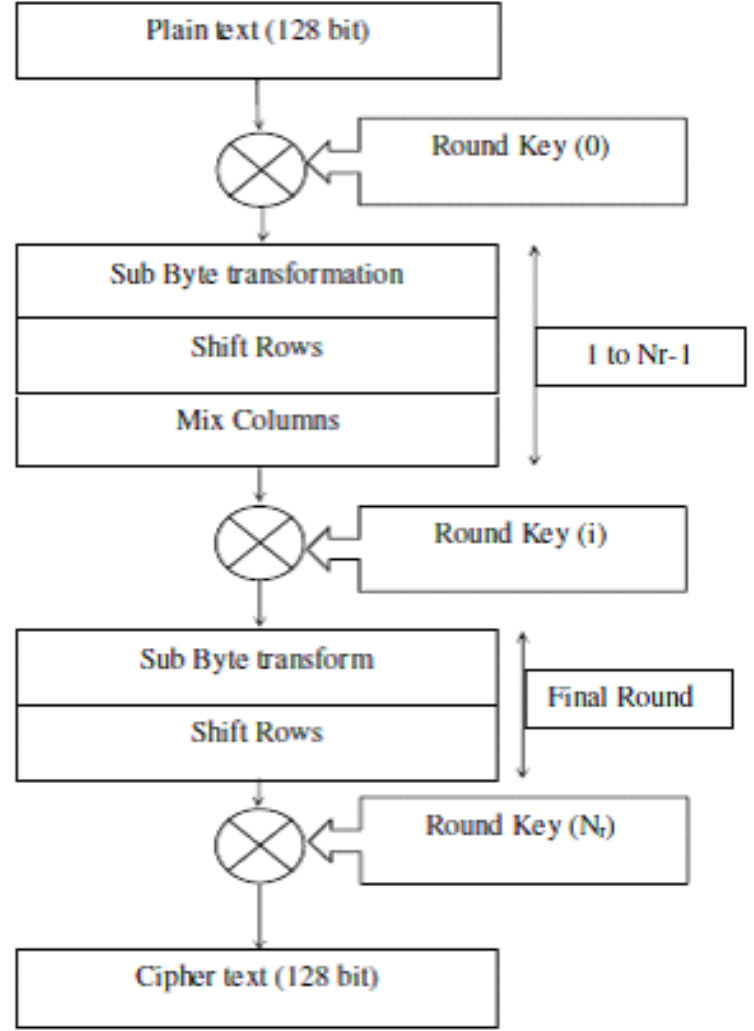

Fig. 1. Algorithms for AES Encryption

The Rijandael deciphering includes four backwards activities of encryptions are acclaim segments of encryptions. They are 1. Backwards Substitutions

2. Backwards Row shifts

3. Backwards Mix Columns

4. Key expansion

\section{A.SubByte and Inverse SubByte change:}

In the Sub Bytes process, each that is override and displace with a Sub Byte using a 8-piece data $\mathrm{n}$ at the Rijndael S-Box. In the opposite Sub Bytes, every byte in figure cross area is supplanted with differentiating opposite Sub Byte. Sub Byte development gives the abnormality figure. The S Box utilized is gotten with the multiplicative opposite finished Galois Field (28) [7], inconceivable without linearity property. Different S-Box execution [7] utilize combinational circuit includes a snake, squarer and unsurprising multipliers. RijndaelS-Box isn't appeared for speed.

\section{B. Move Row Transformation:}

Each area by assured evening out for the opposite side. For AES, the significant portion is missing unaltered. Every byte of to the subsequent line is moved by adjusted side. 
So moreover, $3^{\text {rd }} \& 4^{\text {th }}$ lines are moved by only. Pivot row shift change in like way move activity towards right. Fig.2 depicts the Row shift method.

The tasks of AES Rijndael calculation for encryption what's more, decoding is known as pursues.

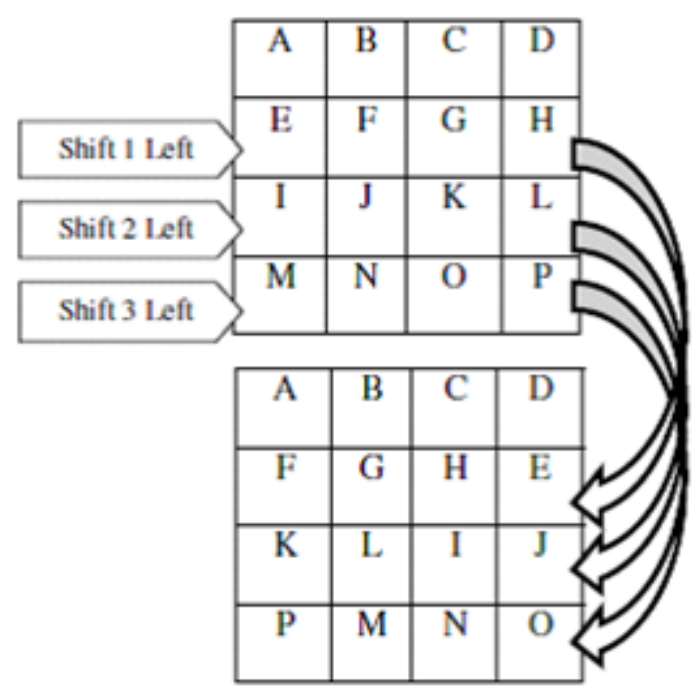

Fig. 2. AES Row shift Operation.

\section{Blend Column and opposite Mix Column activity:}

Mix Columns, the $4^{\text {th }}$ bytes of each condition are joined utilizing a turn around straight change. All sections in the state organize are viewed as a polynomials and it is copied by a fix polynomials. The Mix Columns and opposite Mix Column change are addressed in cross section structure as structure as a condition 1, 2 .

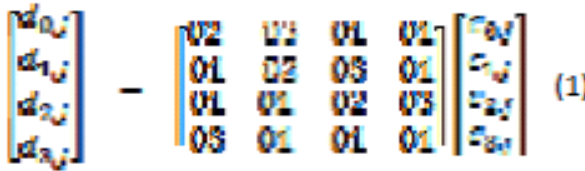

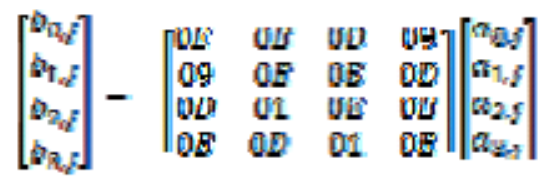

\section{Include around Key activity}

The development, bitwise select activity is perform among yields from Mix Columns and around Key. For AES-128,128 piece XOR endeavors are perform.

\section{PROPOSED WORK}

The projected structure is relied upon to give most unmistakable speed and less zone by map consistent parts of AES to LUTs, ROMs and Block Rams. This model is having three components 1. Key Generation 2. Encryption 3. Disentangling. The AES encryptions and unscrambling center unit contain key age component as a standard unit. This unit give critical key improvement for both encryptions \&Decryptions limits.

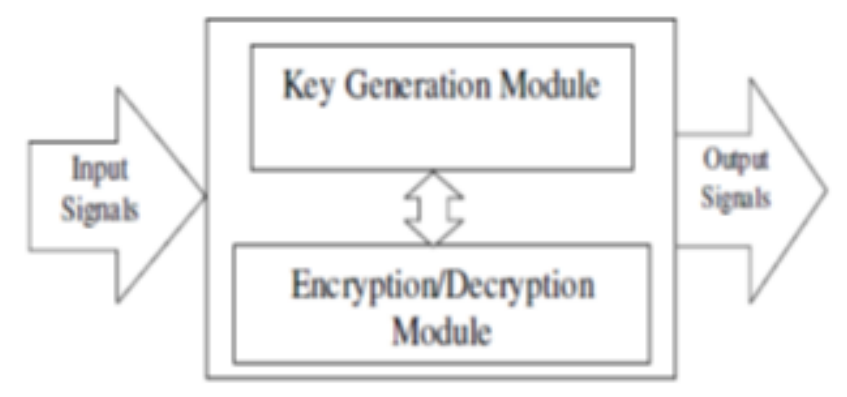

Fig. 3. AES Encryption and Decryption Unit.

The fig shows the square outline of AES Rijndael encryptions and Decryptions from Key production unit as a customary unit. The key age unit incorporates key index of 128bits, S-Box\&XOR doorways for bitwise XOR improvement.

This is depended upon to pass on around keys on each constructive border of to the clock, when it is secured. At any rate in to the projected work, the key age building doesn't require any gear for move action and the port map between key list and S-Box is done by the key move. From this time forward the proposed work offers the incredible circumstance in a domain. In like manner as a projected work the bits are balanced on data course from list to S-Box and surrounding obvious required for each round are managed in ROM and recuperated on each clock. Fig.4 address conventional organizing of key age unit.

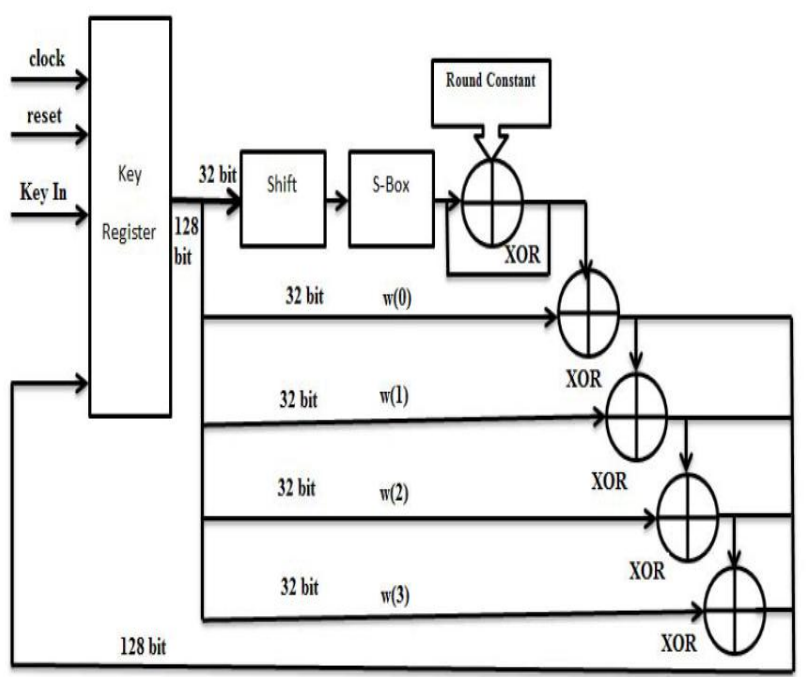

Fig.4 Key Generation Component.

The encryptions unit takes 128 piece substance to be blended and gets around type from type age component to do individually difference in encryption. Fig. 5 displays conventional encryption component.

Published By: 


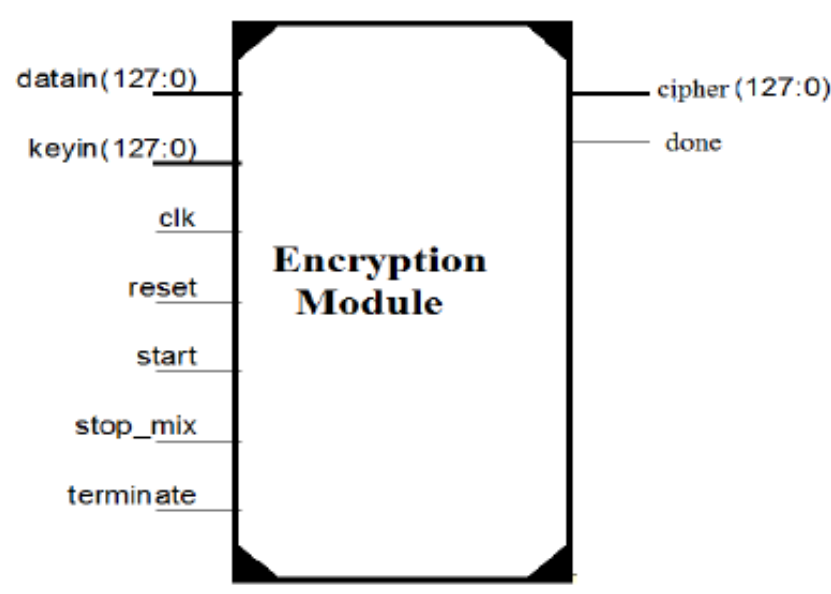

Fig. 5. Encryption Component.

Start, stop mix, end are organize sign passed on through the organize unit. The "done' indication is given to shows encryption is done. Building is as showed up in Fig. 6.

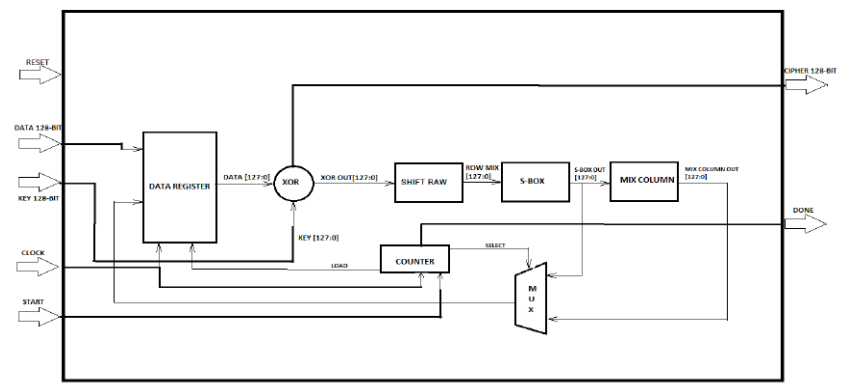

Fig.6 Mix Column Operation is removed in the last stage. To fuse this usefulness proposed configuration.

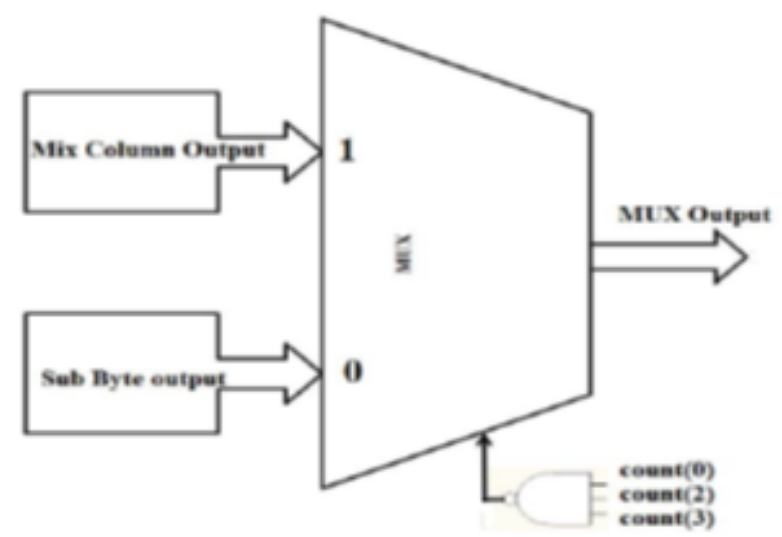

Fig. 7. Hardware to Skip Mix Columns Operation for Last Round.

NAND section \& four-piece oppose are utilized to set \& rearrange decision line of Multiplexers. For check one to ten decision line shall in arrange condition and multiplexers shall pass Mix Columns yield. At any rate on last around, check will be 11 so decision line would rearrange and pass Sub Byte yield.

Move Row action is sifted through to not get any component. Afterwards Curved Significant action information is specified to S-Box through necessary move via port map a sign as shows up by necessary move in Verilog HDL depiction to the game-plan. Since there is no gear for Row shift advancement arrangement gets the upside of territory, power and velocity.

At this projected work, the S-Box was done via a LUT has 8 piece statement ( 256 zones) \& the data sizes of 8 pieces. This execution give difficult through put from the structure by in a general sense decreasing suspension in data way. All things considered the conventional structure takes less number of cuts when disengaged and different combinational system proposed.

The Mix Columns movement of AES contains Galois improvement and four data XOR action. Notwithstanding, not in the smallest degree like combinational use of Galoisfield increment, the projected structure uses ROM base execution of Galois improvement which makes Galois duplication in a general sense faster avoid combinational deferrals.

The unraveling utilize same filtering through a whole structure and take twenty clock cycles to restore the specified figure back to noteworthy substance.

Adjust S-Box masterminding utilizes a general structure of S-Box. Zone of LUT is changed by opposite Sub Byte change. Plan of Decryption component is same as encryptions component with every single approving breaking point of encryptions. Interpreting component contain an additional register for overseeing Round Keys. Consider register is blended B-Ram to spare no of cuts. 'Check' input gives the region of key list a district to be gotten to. The structural design of unscrambling component is appeared in Fig. 8.

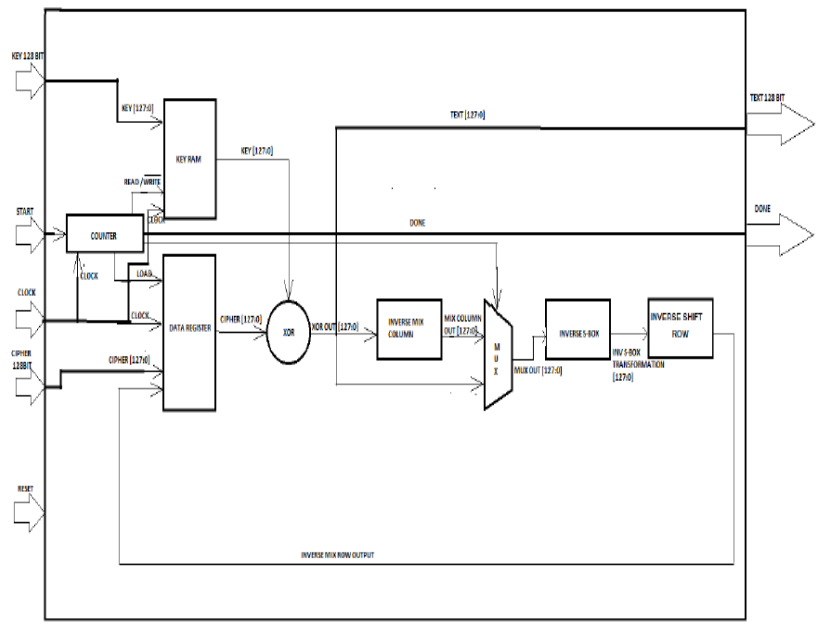

Fig.8 The structural design of unscrambling component

\section{RESULTS}

AES Rijndael figuring is reproduced \& mixed utilize Xilinx 13.1 ISE contraption and the concentrated on has a spot with Virtex-5 family. The approach uses just LUTs, ROMs for the aggregate of the exercises of AES encryptions and unscrambling. Along these lines of reasoning declines contraption use and in a general sense improves the speed stood separated from other execution $[3,4,9]$. The key register in the unscrambling 
component is sifted through as Block-Ram to decrease the proportion of cuts used. The use once-over for contraption 5vlx110tff1136-3 is displayed in Table I.

TABLEI. SLICE LOGIC UTIIIZATION

\begin{tabular}{|l|ll|}
\hline Number of Slice Registers & 128 out of $69120 \quad 0 \%$ \\
\hline Number of Slice LUTs & 1106 out of $69120 \quad 1 \%$ \\
\hline Number used as Logic & 1106 out of 69120 & $1 \%$ \\
\hline
\end{tabular}

Table I. Slice Logic Utilization

In this projected course of action, the encryptions unit take 10 clock cycles to entire the improvement. The most ludicrous link deferment to a game-plan is 3.420ns achieving a biggest repeat of movement as $292.403 \mathrm{MHz}$. The use once-over for contraption $5 \mathrm{vlx} 110 \mathrm{tff} 1136-3$ is existing in Table I.

$$
\text { Throughput }=\frac{\text { Number of block color Frequency }}{\text { Number of Frequency }}
$$

\section{CONCLUSION}

AES-128figuring for encryptions and unraveling is acknowledged. From the arranging of the huge number of activities as LUTs \&ROMs, the projected planning accomplishes a throughputs of $3.74 \mathrm{Gbps}$ and in this way using just $1 \%$ of cuts in the focused on FPGA. Since the velocity is higher than suitably point by point structures, in like manner the proposed game plan fills in as the best snappy encryptions calculation and is from this time forward appropriate for different applications. Also with less region usage, the projected structure can be implanted with other more prominent plans too.

\section{REFERENCES}

1. Abhijith.P.S, Mallika Srivastava, Aparna Mishra, Manish Goswami, B.R.Singh, "High Performance Hardware Implementation of AES Using Minimal Resources", 2013 International Conference on Intelligent Systems and Signal Processing (ISSP).

2. M. Goswami and S. Kannojiya, "High Performance FPGA Implementation of AES Algorithm with 128-Bit Keys,” Proc. IEEE Int Conf. Advances Computing Comm., vol. 1, Himarpur, India, 2011, pp. 281-286.

3. FIPS-197, NIST - National Institute of Standards and Technology, "Announcing the Advanced Encryption Standard (AES)," http://csrc.nist.gov/publications/fips/fips197/fips-197.pdf, 2001.

4. M. Goswami and S. Kannojiya, "High Performance FPGA Implementation of AES Algorithm with 128-Bit Keys,” Proc. IEEE Int. Conf. Advances Computing Comm., vol. 1, Himarpur, India, 2011, pp. 281-286.

5. FIPS-197, NIST - National Institute of Standards and Technology, "Announcing the ADVANCED ENCRYPTION STANDARD (AES)," http://csrc.nist.gov/publications/fips/fips197/fips-197.pdf, 2001.

6. W. Wei, C. Jie and X. Fei, “An Implementation of AES Algorithm on FPGA,” IEEE 9th Int. Conf. on Fuzzy Systems and Knowledge discover 2012, pp. 1615-1617.

7. U. Kretzschmar, A. Astarloa, J. Lazaro, U. Bidarte and J. Jimenez, "Robustness analysis of different AES implementations on SRAM based FPGAs," Int. Conf. on Reconfigurable Computing and FPGAs 2011, pp. $255-260$.

8. J. Daeme and V. Rijmen, "AES proposal: Rijndael," NIST AES Proposal, June 1998.

9. W. Stallings, "Cryptography and network security principles and practice," Pearson edition 2009, pp. 135-160.

10. P.V.S. Shastry, A. Agnihotri, D. Kachhwaha, J. Singh and M.S Sutaone, "A Combinational Logic Implementation of S-Box of AES," IEEE $54^{\text {th }}$ Int. Midwest Symp. on Circuits and Systems (MWSCAS), Aug. 2011, pp. 1-4

\section{AUTHORS PROFILE}

LAXMI PALAMARTHI, Asst.Prof.Department of ECE,Malla Reddy Engineering College for women,Maisammaguda,

Secunderabad,Telangana,India.E-mail:mkn20679@gmail.com.

C.MURALI KRISHNA , Asst.Prof.Department of ECE,Malla Reddy

Engineering College for women,Maisammaguda,

Secunderabad,Telangana,India.E-mail:mkn20679@gmail.com. 